\title{
Mechanics of a curved electrode actuator operating in viscous dielectric media: simulation and experiment
}

\author{
Sindhu Preetham Burugupally \\ Department of Mechanical Engineering, Wichita State University, Wichita, KS
}

67260 USA

\begin{abstract}
Curved electrode electrostatic actuators have been shown to have the potential to manipulate microparticles at high speeds in viscous dielectric media. However, there are no multiphysics models that fully describe the mechanics of these actuators in viscous dielectric media. This work is the first step in the direction of describing the actuator mechanics through Finite Element Method (FEM) simulations and validate it with the experiments. Simulations are performed using the commercial software Coventor MEMS $+{ }^{\circledR}$ and the results are found to be in good agreement with experiments. Through a simple lumped-parameter model of the actuator, we report that the viscous damping effect by the substrate on the actuator submerged in methanol or water media becomes significant for actuation frequencies $>100 \mathrm{~Hz}$.
\end{abstract}

Keywords: Curved electrode electrostatic actuator, Finite element method, Frequency response, Viscous dielectric media, Microelectromechanical systems (MEMS)

\section{Introduction}

Electrostatic actuators are the physical systems comprising of mechanical and electrical components that generate mechanical motion at micron scale $O\left(10^{-6}\right)$ $\mathrm{m}$. These actuators have the potential to physically manipulate microparticles suspended in a viscous media $[1,2,3,4]$. To manipulate microparticles at a fast rate of $O\left(10^{2}\right)$ particles/s, there is a need for a high speed actuator that can oscillate at a frequency $O\left(10^{2}\right) \mathrm{Hz}$ and achieve a displacement on the order of few microns in the viscous microenvironment. The most common electrostatic actuator configurations such as parallel plate [2] and comb-type [5] have shortcomings - the requirement of large actuation voltages in comb drives [6] and the displacement limitation in parallel plates [7]. To overcome these shortcomings, a curved electrode electrostatic actuator in a clamped-clamped beam configuration was designed that operates in a stable manner above the pull-in voltage and achieves large displacement at low actuation voltages (Fig. 1) [8]. The curved electrode actuators have been experimentally shown to have the potential to manipulate microparticles at high speeds in viscous dielectric media [9]. However, there are no multiphysics models that fully describe the mechanics of these actuators in the viscous dielectric media. An in-depth understanding 


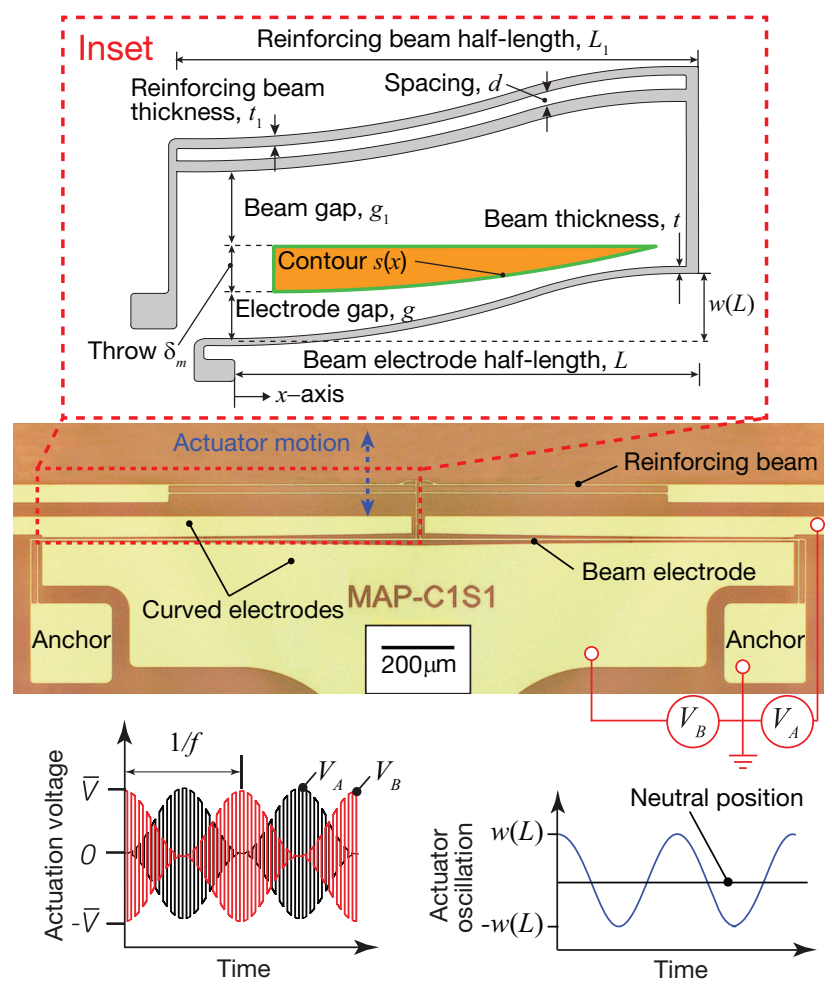

Figure 1: A stereomicroscope image of the actuator with actuation voltage waveforms $\left(V_{A}, V_{B}\right)$ and displacement signal $(w(L))$ shown. The inset shows a portion of the actuator with geometric parameters. The actuator comprises of a beam electrode, set of curved electrodes, and a reinforcing beam. The beam electrode and reinforcing beam together form the compliant clamped-clamped structure. The curved electrodes are immovable rigid members whose contour $s(x)=\delta_{m}(x / L)^{2}$. See Table 1 for the more details.

of the actuator mechanics in the viscous dielectric media is very beneficial, especially for designing actuators meant for biological applications such as cell probing and sorting $[2,8,10]$.

In this work, we analyze the mechanics of the curved electrode actuator in viscous dielectric media for the nonzipped operation mode, where the beam electrode does not come in physical contact with the curved electrode. Here, the actuator performance is analyzed in terms of displacement and frequency range through Finite Element Method (FEM) simulations and later validated with experiments. FEM simulations are performed using Coventor MEMS+ ${ }^{\circledR}$ and found to be in good agreement with the experiments. In addition, a simple lumped-parameter model of the actuator is derived to analyze the effect of viscous damping by the substrate on the actuator. This work is the first step in 
Table 1: Nominal and measured actuator parameters and physical parameters. Measured parameters given as mean \pm standard deviation for four measurements.

\begin{tabular}{lc}
\hline Parameter & Numerical value \\
\hline Beam electrode half-length, $L$ & $1000 \mu \mathrm{m}$ \\
Reinforcing beam half-length, $L_{1}$ & $670 \mu \mathrm{m}$ \\
Beam electrode thickness, $t$ & $4.7 \pm 0.2 \mu \mathrm{m}$ \\
Reinforcing beam thickness, $t_{1}$ & $3.8 \pm 0.2 \mu \mathrm{m}$ \\
Electrode gap, $g$ & $3.5 \pm 0.1 \mu \mathrm{m}$ \\
Substrate clearance, $g_{o}$ & $3 \mu \mathrm{m}$ \\
Minimum beam gap, $g_{1}$ & $30 \mu \mathrm{m}$ \\
Reinforcing beam spacing, $d$ & $17 \mu \mathrm{m}$ \\
Beam electrode height, $h$ & $45 \pm 0.4 \mu \mathrm{m}$ \\
Throw of the curved electrode, $\delta_{m}$ & $10 \mu \mathrm{m}$ \\
Overall actuator stiffness, $k_{e}$ & $10 \mathrm{~N} / \mathrm{m}$ \\
Young's modulus of Si [11], $E$ & $130 \mathrm{GPa}$ \\
Relative permittivity of $\mathrm{Al}_{2} \mathrm{O}_{3}$ & 9 \\
\hline
\end{tabular}

understanding the effect of viscous media on actuator performance characterized in terms of the cutoff frequency. The following sections describe the actuator operation, model and experiment setup, and results that describe the actuator behavior in viscous environments where the quality factor $(Q)$ is at least two orders of magnitude lower than in air.

\section{Materials and Methods}

The curved electrode actuator shown in Figs. 1 and 2 is fabricated on a 100 mm diameter silicon-on-glass (SOG) wafer (purchased from PlanOptik, Elsoff, Germany) made by anodic bonding of a silicon wafer $(<100>$ p-type boron doped to $100 \mathrm{ppm}$ ) to a $500 \mu \mathrm{m}$ thick Borofloat33 glass wafer and then thinning and polishing the silicon wafer to a thickness of $45 \mu \mathrm{m}$. The actuator components are micromachined from the silicon layer with parameters given in Table 1, while the glass layer acts as a handling substrate and provides optical access for viewing with an inverted microscope. The actuator fabrication details are presented in the Appendix section (Fig. A1).

The actuator is characterized in terms of actuator displacement amplitude $w(L)$ at different voltage amplitudes $\bar{V} \in[1,4] \mathrm{V}$ in the increments of $1 \mathrm{~V}$ and actuation frequencies $f \in[0,4096] \mathrm{Hz}$ using the test setup shown in Fig. 3. For the static tests, the actuator is supplied with a high frequency $(500 \mathrm{kHz})$ 
Table 2: Nominal viscous dielectric media properties.

\begin{tabular}{ll}
\hline Parameter & Value \\
\hline Relative permittivity of deionized water & 80 \\
Relative permittivity of methanol & 32 \\
Relative permittivity of silicone oil & 2.6 \\
Relative permittivity of hypothetical fluid & 1 \\
Density of deionized water $\left(\mathrm{kg} / \mathrm{m}^{3}\right)$ & 1000 \\
Density of methanol $\left(\mathrm{kg} / \mathrm{m}^{3}\right)$ & 800 \\
Density of silicone oil $\left(\mathrm{kg} / \mathrm{m}^{3}\right)$ & 900 \\
Density of hypothetical fluid $\left(\mathrm{kg} / \mathrm{m}^{3}\right)$ & 900 \\
Dynamic viscosity of deionized water $(\mathrm{Pa}-\mathrm{s})$ & 0.00089 \\
Dynamic viscosity of methanol $(\mathrm{Pa}-\mathrm{s})$ & 0.00054 \\
Dynamic viscosity of silicone oil $(\mathrm{Pa}-\mathrm{s})$ & 0.00459 \\
Dynamic viscosity of hypothetical fluid $(\mathrm{Pa}-\mathrm{s})$ & 0.00459
\end{tabular}

square wave voltage signal at different voltage amplitudes $\left(V_{A}\right.$ or $\left.V_{B}=\bar{V}\right)$ to one set of the curved electrodes located on one side of the beam electrode. For the dynamic tests, the actuator is supplied with two amplitude-modulated voltage signals $V_{A}(t)=\frac{1}{2} \bar{V}(1+\cos 2 \pi f t) \Pi(t)$ and $V_{B}(t)=\frac{1}{2} \bar{V}(1-\cos 2 \pi f t) \Pi(t)$, where $\cos 2 \pi f t$ and $-\cos 2 \pi f t$ are the modulation waveforms, $t$ is the time, $\Pi(t)=\frac{4}{\pi} \sum_{j=1}^{\infty} \frac{1}{2 j-1}(-1)^{j-1} \cos 2 \pi f_{c} j t$, and $f$ and $f_{c}(500 \mathrm{kHz})$ are the modulation (also called actuation) and carrier frequencies, respectively (Fig. 1). Note that the voltages $V_{A}$ and $V_{B}$ are $180^{\circ}$ out-of-phase to drive the actuator in forward and backward directions. For given actuation voltages $V_{A}$ and $V_{B}$, the cinephotomicrography was recorded at frame rates greater than 6 times the corresponding actuation frequency $f$ so as to prevent aliasing. A custom image processing MATLAB ${ }^{\circledR}$ script is used to create time traces of the actuator displacement for follow-up analyses of frequency content and peak-to-peak displacement amplitude $2 w(L)$.

The 3-dimensional Finite Element Method (FEM) model of the actuator is built and analyzed using the commercial software Coventor MEMS+ ${ }^{\circledR}$ comprising of mechanical and electrical submodels (Fig. 2b). Here, the beam electrode, curved electrode, and reinforcing beam are individually constructed from 120 Bernoulli beam elements. Each beam element has 6 nodes, where each node has one degree of freedom that corresponds to the node displacement in the $x-y$ plane with direction perpendicular to the beam element length. To determine the appropriate number of beam elements, a convergence test is performed on the actuator model; here, first an FEM model of the actuator with a different number of beam elements ranging from 20 to 200 is constructed, then a fixed 
(a)

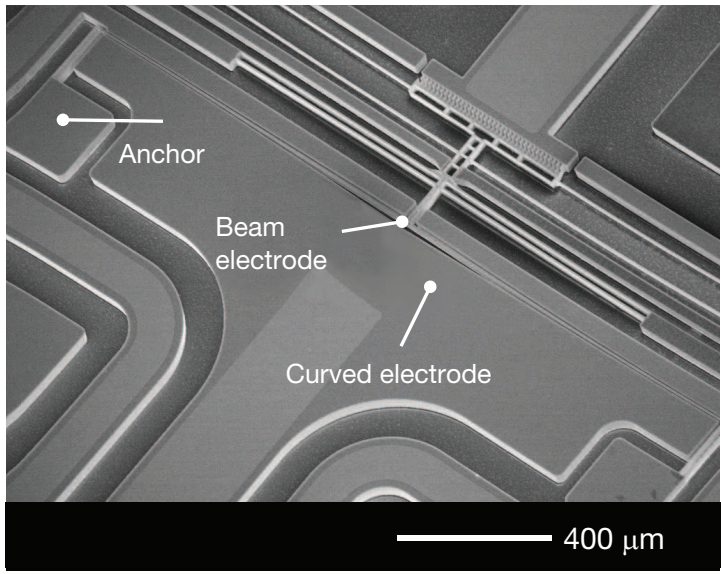

(b)

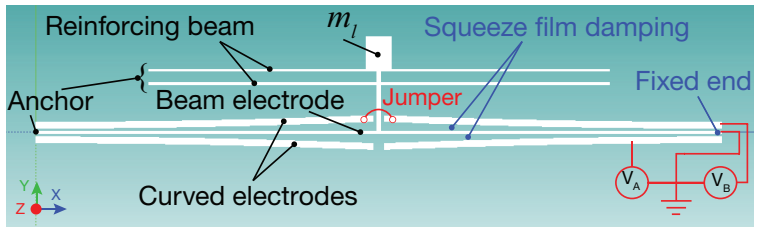

(c)

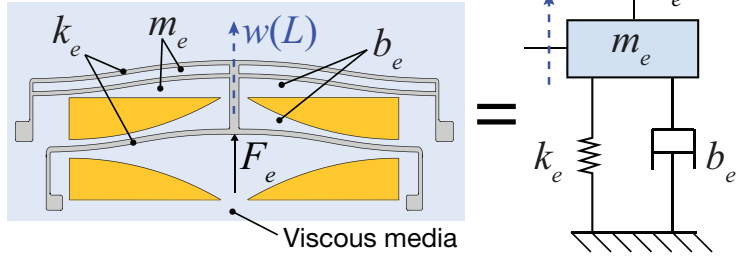

Figure 2: Curved electrode actuator. (a) A scanning electron microscope (SEM) image of the actuator. (b) A 3-dimensional FEM model of the actuator built in Coventor MEMS+ ${ }^{\circledR}$ software. (c) A schematic sketch of the actuator and its equivalent lumped model.

DC voltage $(4 \mathrm{~V})$ is applied to one set of the curved electrodes located on one side of the beam electrode, and corresponding static displacements $w(L)$ of the actuator are obtained (Fig. A2). Based on the convergence test (Fig. A2), in this work, we chose 120 beam elements to construct the individual actuator components. The model does not include the viscous fluid damping by substrate. The inertial loading effect by the viscous media trapped between the reinforcing beams is incorporated into the model - as a lumped mass $m_{l}$ made of silicon - located at the midpoint of the reinforcing beam (Fig. 2b). To prevent electrical shorting between the beam electrode and curved electrode, a $20 \mathrm{~nm}$ thick $\mathrm{Al}_{2} \mathrm{O}_{3}$ insulator film is added to the curved electrodes. To reduce the computation burden and assist in the solution convergence, the electrostatic 

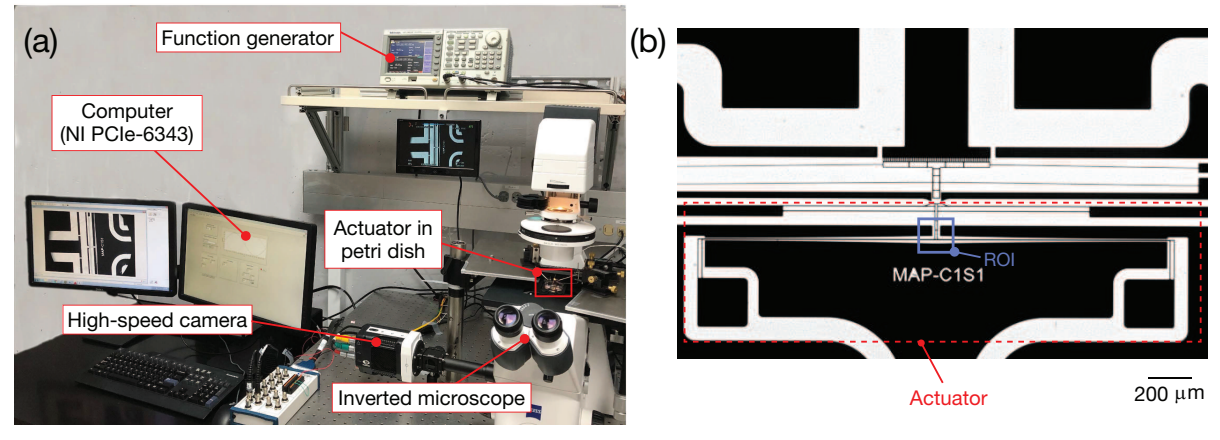

Figure 3: (a) Actuator evaluation experiment setup. (b) A photomicrograph of the actuator submerged in viscous media (deionized water) captured using high-speed camera and inverted microscope. The test setup comprises of an inverted microscope (Zeiss Observer A1 microscope, Germany) and high-speed camera (Miro M110, Vision Research, USA) for photomicrography of the moving actuator in the region of interest (ROI), and LabVIEW/NI PCIe-6343 data acquisition system and function generator (Tektronix AFG3022C, Beaverton, OR, USA) for triggering the high-speed camera to initiate cinephotomicrography and generating two amplitude-modulated voltage waveforms $V_{A}$ and $V_{B}$.

and fluidic (squeeze film damping) forces are linearized; here, the linearization value is set to $0.25 \mu \mathrm{m}$. The source code for simulating the actuator mechanics is made available on GitHub [14].

A lumped-parameter model of the actuator operating in the viscous dielectric media is derived using the Newton's second of motion (Fig. 2c). The actuator is idealized as a spring-mass-damper system with a single degree-of-freedom. The frequency response of the actuator is given by the transfer function $T$ [15]

$$
T=\frac{F_{e}}{\sqrt{\left(k_{e}-4 m_{e} \pi^{2} f^{2}\right)^{2}+\left(2 \pi f b_{e}\right)^{2}}}
$$

where $k_{e}$ is the overall stiffness, $m_{e}$ is the effective mass [16], $b_{e}$ is the overall damping coefficient [17], and $F_{e}$ is the amplitude of the electrostatic force acting on the actuator. The mathematical expressions for these terms are given by 


$$
\begin{aligned}
m_{e}=2 \rho_{f} h\left(0.398 d L_{1}+\frac{\pi}{2} h\left(L_{1}+L\right)\right) \\
\quad+0.796 \rho h\left(t L+2 t_{1} L_{1}\right) \\
b_{e}=\left[\frac{4 \mu_{f} h^{3} L}{(g+s(x)-w(x))^{3}}+\frac{4 \mu_{f} h^{3} L_{1}}{\left(g_{1}-w(x)\right)^{3}}\right] \Gamma \\
k_{e}=\frac{2 E h t^{3}}{L^{3}}+\frac{4 E h t_{1}^{3}}{L_{1}^{3}} \\
F_{e}=\frac{\epsilon_{0} \epsilon_{f} L h \bar{V}^{2}}{(g+s(x)-w(x))^{2}}
\end{aligned}
$$

where $\mu_{f}$ is the dynamic viscosity of the media, $w(x)$ is the displacement of the beam electrode or reinforcing beam at any location $x$ along the beam length, $\epsilon_{f}$ is the relative permittivity of the media, and $\rho_{f}$ and $\rho$ are the densities of the media and silicon, respectively. Note the first parenthesis term of the effective mass $m_{e}$ (Eq. 2a) corresponds to the inertial effect of the media, while the second parenthesis term corresponds to the effective mass of the beam electrode and reinforcing beam. The square parenthesis term of the damping coefficient $b_{e}$ models the squeeze film damping by the media on the actuator (Eq. 2b). The proximity of the actuator to the glass substrate results in the additional damping on the actuator, and is modeled by $\Gamma$, where $\Gamma=\frac{4 g^{3} t+2 g_{o}^{3} h}{g^{3} t+2 g_{o}^{3} h}[17]$. Here, $g_{o}$ is the clearance between the actuator and glass substrate. In the nonzipped mode of operation, the actuator displacement is small $(<5 \mu \mathrm{m})$ and hence the overall damping coefficient $b_{e}$ and electrostatic force $F_{e}$ are linearized. The numerical values of the media properties are given in Table 2.

\section{Results and discussion}

Through FEM model and experiment, we analyze the mechanics of the actuator in the nonzipped operation. The nonzipped operating mode occurs for voltages below the pull-in voltage, and found to be around $4 \mathrm{~V}$ for deionized water media [18]. The actuator displacement $w(L)$ increases with actuation voltage amplitude $\bar{V}$ (Fig. 4). The actuator achieves displacement $w(L)$ on the order of few microns at low actuation voltage with high displacement sensitivity $(\sim 0.28$ $\mu \mathrm{m} / \mathrm{V})$. The static displacement of the actuator in deionized water media for actuation voltages $\bar{V} \in[1,4] \mathrm{V}$ predicted using the FEM model is in good agreement with the experiment data collected from three actuators (Fig. 4). Similarly, the dynamic response of the actuator obtained using the simulation for deionized water media at low actuation voltage $(\bar{V}=4 \mathrm{~V})$ and low actuation frequency $(f=1 \mathrm{~Hz})$ agrees well with the experiment (Fig. 5).

The FEM simulation results are compared with the experimentally obtained frequency response plots in the nonzipped mode for deionized water and methanol media (Fig. 6). Since deionized water has higher relative permittivity than methanol (Table 1), the actuator peak-to-peak displacements in deionized 


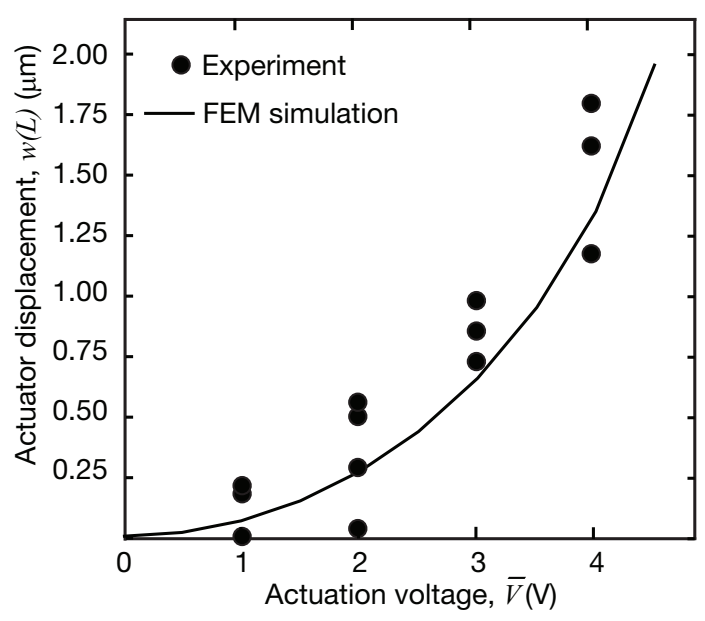

Figure 4: Comparison of actuator static displacement amplitude $w(L)$ in deionized water media for different actuation voltage amplitudes $\bar{V}$ obtained using the FEM model and experiment. The displacement measurement has a quantization error of $\pm 1 / 2$ pixel $= \pm 0.15 \mu \mathrm{m}$.

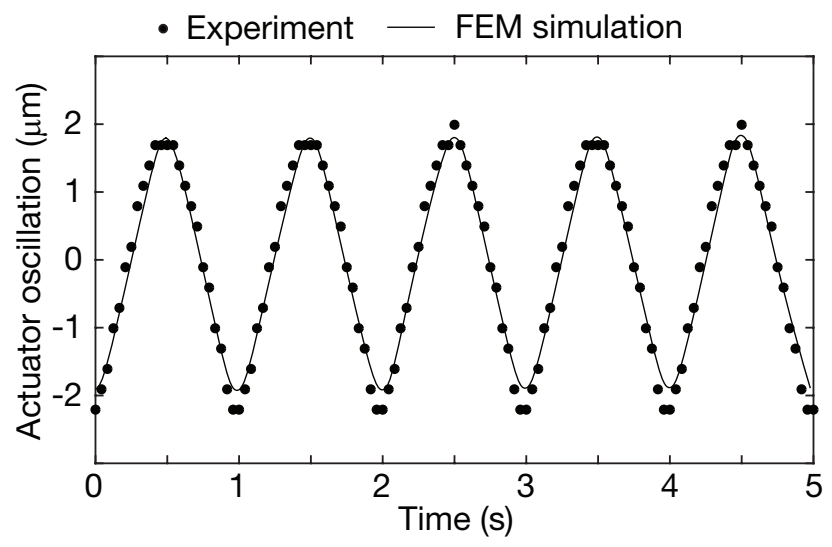

Figure 5: Measured and simulated displacement-time trace of the actuator in deionized water media over five successive cycles for $\bar{V}=4 \mathrm{~V}$ and $f=1 \mathrm{~Hz}$. The displacement measurement has a quantization error of $\pm 1 / 2$ pixel $= \pm 0.15$ $\mu \mathrm{m}$. 


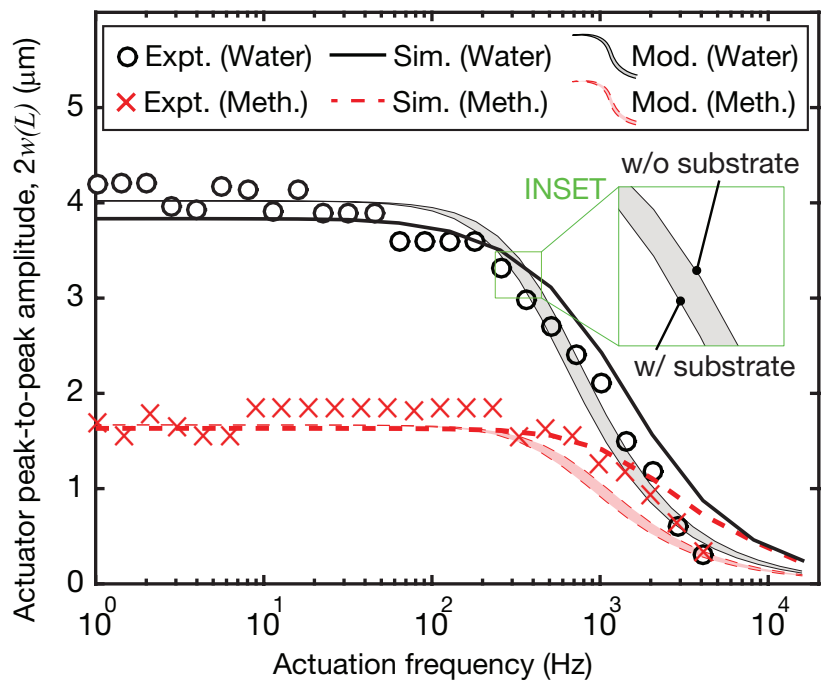

Figure 6: Measured and simulated frequency response of the actuator in deionized water and methanol media for $\bar{V}=4 \mathrm{~V}$. The FEM model is labeled as Sim., while the lumped model is labeled as Mod. Here, the actuator operates in the nonzipped mode where the peak-to-peak displacement amplitudes are less than $5 \mu \mathrm{m}$. Inset shows the frequency response of the actuator with (w/ substrate) and without (w/o substrate) considering the viscous damping effect by the substrate. The displacement measurement has a quantization error of $\pm 1 / 2$ pixel $= \pm 0.15 \mu \mathrm{m}$.

water are higher for a given voltage amplitude $\bar{V}$ due to the stronger electrostatic force. The measured and simulated frequency responses of the actuator for both media show similar trends - the actuator displacements begin to roll-off beyond $300 \mathrm{~Hz}$ actuation frequency. For actuation frequencies higher than 100 $\mathrm{Hz}$, the actuator displacement values obtained from the FEM model begin to deviate from the experiment. This is due to the increase in difference between the actual and simulated damping force on the actuator. As earlier mentioned, the viscous fluid damping on the actuator by the substrate is neglected in the FEM model, while in reality (experiment), the actuator experiences this viscous damping force. Hence, the FEM model gravitates towards over predicting the actuator displacement and frequency bandwidth (Fig. 6). This inference has been verified by modeling the actuator as a lumped model whose transfer function is given by Eq. 1. As shown in Fig. 6, the actuator modeled without the viscous damping by the substrate (w/o substrate) has slightly higher displacement compared to the actuator modeled with the viscous damping by the substrate (w/ substrate). In contrast, for actuation frequency $f<100 \mathrm{~Hz}$, the effect of viscous damping by the substrate on the actuator is negligible.

A proper choice of media characterized in terms of dielectric constant (relative permittivity) and dynamic viscosity is essential for the optimal performance 


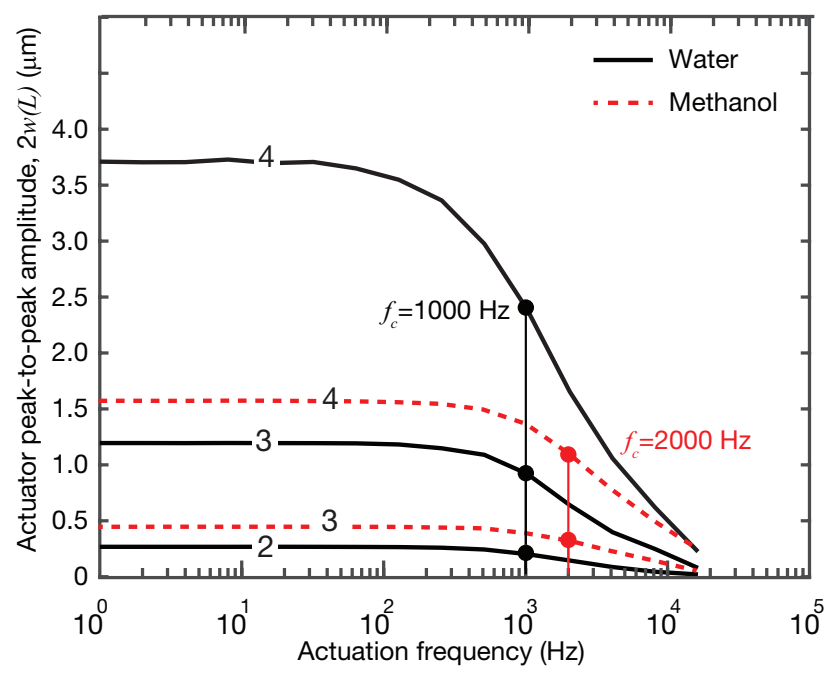

Figure 7: Simulated frequency response of the actuator in deionized water and methanol for different voltages $\bar{V} \in\{2,3,4\}$ using the FEM model.

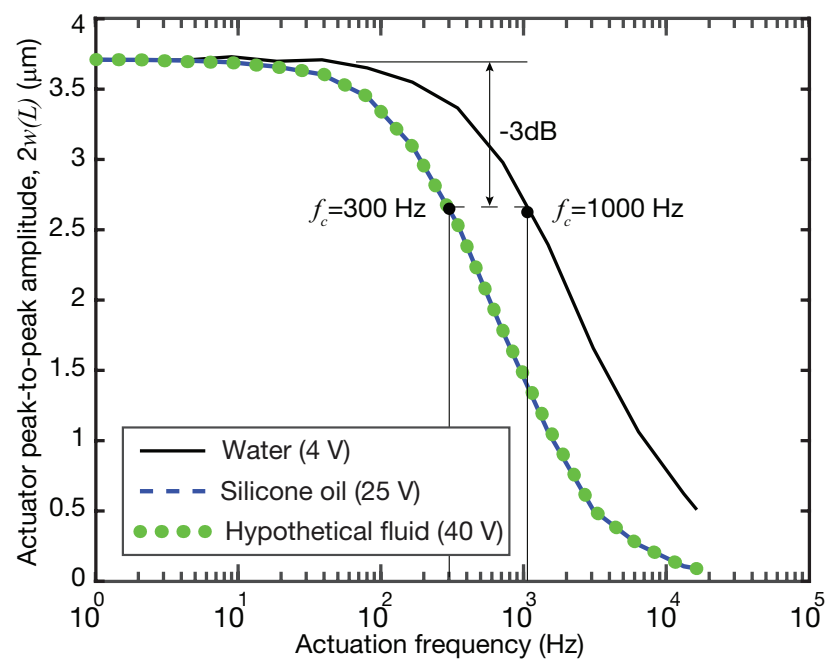

Figure 8: Simulated frequency response of the actuator in different media using the FEM model. Here, the actuation voltages are chosen such that the peakto-peak actuator displacements at $1 \mathrm{~Hz}$ are equal $(3.7 \mu \mathrm{m})$. The hypothetical fluid has a relative permittivity of the air and dynamic viscosity of the silicone oil. This presents a scenario where the media is a high viscous fluid with low permittivity. See Table 2 for fluid properties.

of the actuator. Different media namely, silicone oil, water, methanol, and hy- 
pothetical fluid are investigated to understand the effect of the media properties on actuator performance (Figs. 7 and 8, Table 2). For a given media and low actuator displacements, the cutoff frequency of the actuator is found to be independent of the actuation voltage and/or actuator displacement amplitude (Fig. 7). For instance, the cutoff frequency in water is $1000 \mathrm{~Hz}$ for voltages $\bar{V}=2,3$, and $4 \mathrm{~V}$. This implies the low actuator displacements $(<5 \mu \mathrm{m})$ will not result in invoking the nonlinear effect of the squeeze film damping that can affect the actuator's cutoff frequency. An actuator immersed in a high viscous media will have higher squeeze film damping and a lower cutoff frequency. For instance, the cutoff frequency $f_{c}=1000 \mathrm{~Hz}$ reduces to $f_{c}=300 \mathrm{~Hz}$ when the media is changed from water to silicone oil (Fig. 8).

\section{Conclusions}

Currently, there are no multiphysics models that fully describe the mechanics of the curved electrode actuators submerged in the viscous dielectric media. In this paper, we developed an FEM model of the actuator using a commercial simulation software Coventor MEMS ${ }^{\circledR}$, and validated the model using experiments for the nonzipped operating mode. The simulation results are found to be in good agreement with experiments. Two observations are reported in this study: (1) for a given media and low actuator displacements, the cutoff frequency of the actuator is independent of the actuation voltage and/or actuator displacement; and (2) an actuator immersed in a high viscous media will have higher squeeze film damping and a lower cutoff frequency. Through a simple lumped-parameter model of the actuator, we report that the viscous damping effect by the substrate on the actuator submerged in methanol or water media becomes significant for actuation frequencies $>100 \mathrm{~Hz}$.

\section{Appendix}

Actuator Fabrication Recipe: On the SOG wafer, a composite metal film comprising of $30 \mathrm{~nm} \mathrm{Cr}$ and $200 \mathrm{~nm} \mathrm{Au}$ is deposited on the silicon side by ebeam evaporation and patterned by photolithography to make electrical contact pads (a, b). Next, an etch mask to be used for bulk micromachining of actuator components is defined by depositing a $1000 \mathrm{~nm}$ thick $\mathrm{SiO}_{2}$ layer using a plasma enhanced chemical vapor deposition (PECVD) process (processed at $250{ }^{\circ} \mathrm{C}$ using $\mathrm{SiH}_{4} / \mathrm{N}_{2} \mathrm{O}$ gases), patterned using a photoresist mask, and then etched using inductively coupled plasma assisted reactive ion etching process (RIEICP; using $\mathrm{CHF}_{3} / \mathrm{Ar}$ gases) (c, d). The high-aspect ratio actuator features (curved electrodes, beam electrode, reinforcing beam) are machined by DRIE using a standard Bosch process in 5s:2s step cycles; the glass substrate acts as an etch stop (e). After DRIE, the movable structures of the actuator are released by timed $\mathrm{HF}\left(49 \% \mathrm{HF}: \mathrm{H}_{2} \mathrm{O}:: 1: 1\right.$ volume ratio) wet etch process for approximately 2 minutes at standard room temperature, triple rinsed in water, 


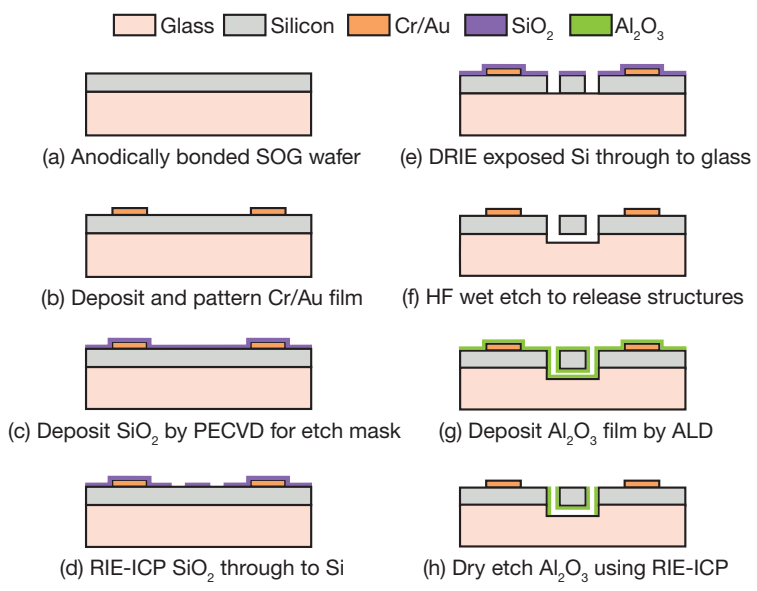

Figure A1: Fabrication scheme of the actuator. [Figure adapted from Ref. [8] with permission from Springer Nature Microsyst. Technol. 24, 3479 (2018). Copyright 2018 Springer-Verlag GmbH Germany, part of Springer Nature.]

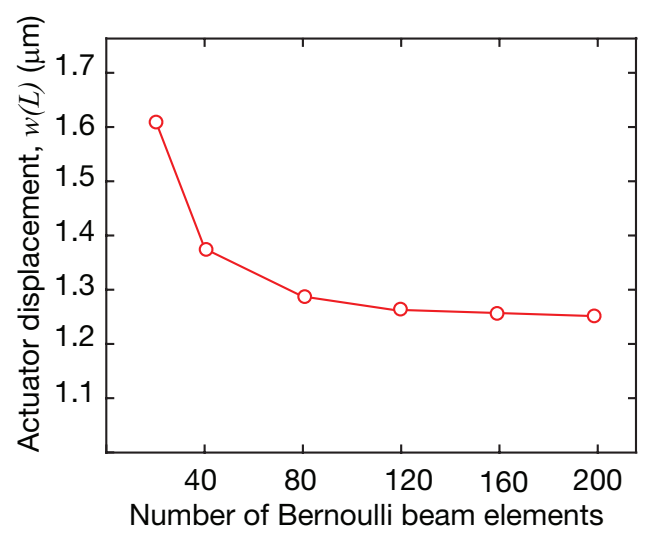

Figure A2: A convergence plot for the nonzipped operating mode of the actuator for $\bar{V}=4 \mathrm{~V}$ in deionized water media. The convergence test shows that convergence begin to occur for Bernoulli beam elements $\geq 120$; hence 120 beam elements are chosen for constructing the 3-dimensional FEM model of the actuator.

then in methanol, and then dried at room temperature (f). Next, the actuator is coated with $10 \mathrm{~nm}$ thick $\mathrm{Al}_{2} \mathrm{O}_{3}$ insulator film using atomic layer deposition (ALD) process (processed at $120{ }^{\circ} \mathrm{C}$ using TMA $/ \mathrm{O}_{2}$ precursors) (g). Finally, the $10 \mathrm{~nm}$ thick $\mathrm{Al}_{2} \mathrm{O}_{3}$ insulator film is anisotropically etched using inductively coupled plasma assisted reactive ion etching process (RIE-ICP; using $\mathrm{BCl}_{3} / \mathrm{Cl}_{2}$ gases) to unmask the $\mathrm{Au} / \mathrm{Cr}$ electrical contact pads that were coated with $\mathrm{Al}_{2} \mathrm{O}_{3}$ during the ALD process (h). 


\section{Acknowledgements}

The author would like to thank David Hoelzle at The Ohio State University for granting access to research facilities to conduct experiments shown in this work. Also, the author would like to that Coventor MEMS $+{ }^{\circledR}$ for providing the software application. This work was supported in part by University of Notre Dame Walther Cancer Foundation IITP Fellowship, University Research/Creative Projects award at Wichita State, and Wichita State and Notre Dame capitalization funds. Technical consultation was given by the Notre Dame Nanofabrication Facility.

\section{References}

[1] S. Yang, Q. Xu, Journal of Micro-Bio Robotics 13(1), 1 (2017). DOI 10.1007/s12213-017-0098-2. URL https://doi.org/10.1007/s12213017-0098-2

[2] S. Warnat, H. King, C. Forbrigger, T. Hubbard, Journal of Micromechanics and Microengineering 25(2), 025011 (2015). URL http://stacks.iop.org/0960-1317/25/i=2/a $=025011$

[3] Z.L. Shen, M.R. Dodge, H. Kahn, R. Ballarini, S.J. Eppell, Biophysical Journal 95(8), $3956 \quad$ (2008). $\quad$ DOI https://doi.org/10.1529/biophysj.107.124602. URL http://www.sciencedirect.com/science/article/pii/S0006349508785345

[4] D. Desmaële, M. Boukallel, S. Régnier, Journal of Biomechanics 44(8), 1433 (2011). DOI https://doi.org/10.1016/j.jbiomech.2011.02.085. URL http://www.sciencedirect.com/science/article/pii/S0021929011001771

[5] N. Scuor, P. Gallina, H.V. Panchawagh, R.L. Mahajan, O. Sbaizero, V. Sergo, Biomedical Microdevices 8(3), 239 (2006). DOI 10.1007/s10544006-8268-3. URL https://doi.org/10.1007/s10544-006-8268-3

[6] D.J. Bell, T.J. Lu, N.A. Fleck, S.M. Spearing, Journal of Micromechanics and Microengineering 15(7), S153 (2005). URL http://stacks.iop.org/0960$1317 / 15 / \mathrm{i}=7 / \mathrm{a}=022$

[7] S.D. Senturia, Microsystem Design (Springer US, 2001)

[8] S.P. Burugupally, J.A. Mangels, Microsystem Technologies 24(8), 3479 (2018). $\quad$ DOI 10.1007/s00542-018-3751-3. URL https://doi.org/10.1007/s00542-018-3751-3

[9] S.P. Burugupally, D. Hoelzle, Applied Physics Letters 113(7), 074102 (2018). DOI 10.1063/1.5042456. URL https://doi.org/10.1063/1.5042456 
[10] D.J. Hoelzle, C.K. Chan, M.B. Scott, M.A. Lake, A.C. Rowat, Journal of Applied Physics 117(1), 014503 (2015). DOI 10.1063/1.4905385. URL http://dx.doi.org/10.1063/1.4905385

[11] M.A. Hopcroft, W.D. Nix, T.W. Kenny, Journal of Microelectromechanical Systems 19(2), 229 (2010)

[12] K.R. Williams, K. Gupta, M. Wasilik, Journal of Microelectromechanical Systems 12(6), 761 (2003). DOI 10.1109/JMEMS.2003.820936

[13] V. Mukundan, B.L. Pruitt, Journal of Microelectromechanical Systems 18(2), 405 (2009). DOI 10.1109/JMEMS.2009.2013398

[14] S.P. Burugupally. Curved electrode electrostatic actuator simulation in DI water and methanol media (2018). DOI 10.5281/zenodo.1482724. URL https://doi.org/10.5281/zenodo.1482724.

[15] L. Meirovitch, Fundamentals of Vibrations. McGraw-Hill higher education (McGraw-Hill, 2001)

[16] A. Rollier, M. Faucher, B. Legrand, D. Collard, L. Buchaillot, CoRR abs/0711.3321 (2007). URL http://arxiv.org/abs/0711.3321

[17] R.M. Shih, D.S. Contreras, T.L. Massey, J.T. Greenspun, K.S.J. Pister, in 2018 IEEE Micro Electro Mechanical Systems (MEMS) (2018), pp. 596599. DOI 10.1109/MEMSYS.2018.8346624

[18] B.S. Preetham, M.A. Lake, D.J. Hoelzle, Napa Microsystems Workshop $2017(2017)$ 